\title{
Tecnologías digitales para el estudio, la difusión y preservación del patrimonio histórico y cultural gallego: contribuciones del proyecto Epigraphica 3.0*
}

\author{
Digital Technologies for the Study, Dissemination and Preservation of the Galician Historical \\ and Cultural Heritage: Contributions from Epigraphica 3.0 Project
}

\author{
DAVID ESPINOSA ESPINOSA ${ }^{1}$ \\ davidespinosaespinosa@gmail.com \\ MIGUEL CARRERO PAZOS ${ }^{2}$ \\ miguel.carrero.pazos@gmail.com
}

\section{RESUMEN}

Este trabajo tiene por objeto dar a conocer las contribuciones del proyecto «Epigraphica 3.0: Hacia la creación y diseño de un corpus digital de inscripciones latinas de la provincia de Ourense» en materia de documentación, estudio y difusión del patrimonio epigráfico gallego. Para ello se presenta un conjunto de experiencias y prácticas de investigación que están siendo desarrolladas en la actualización, revisión y socialización del fondo epigráfico romano de la citada provincia. El resultado es un corpus online y en open access que utiliza la fotogrametría Structure from Motion para el registro de las inscripciones, y una serie de técnicas de tratamiento de imagen digital para el análisis epigráfico y la restitución textual. El objetivo final es tratar de paliar algunos de los efectos negativos que determinados problemas estructurales de Galicia, caso del despoblamiento rural y el envejecimiento demográfico, están ocasionando en su preservación y conocimiento.

Palabras clave: Galicia; Epigrafía romana; patrimonio cultural; tecnologías digitales; Web 3.0.

\section{Abstract}

This work aims to present the contributions of the project «Epigraphica 3.0: Towards a digital corpus of Latin inscriptions from Ourense province» for the recording, study and dissemination of the

* Los autores quisieran expresar su gratitud a cuantas personas e instituciones están haciendo posible este proyecto. Asimismo a los evaluadores del artículo, quienes han contribuido con sus comentarios a la versión definitiva del texto. David Espinosa Espinosa y Miguel Carrero Pazos son beneficiarios de sendos contratos de investigación postdoctoral financiados por la Xunta de Galicia (Plan I2C, modalidades B y A, respectivamente).

1 Grupo de Investigación "Síncrisis. Investigación en Formas Culturais" (GI-1919) y Grupo de Investigación "Ciudades Romanas" (UCM/930692/HIST). Universidade de Santiago de Compostela. https://orcid.org/0000-0002-2337-9047.

2 "Grupo de Estudios para la Prehistoria del NO Ibérico. Arqueología, Antigüedad y Territorio" (GI1534). Universidade de Santiago de Compostela. https://orcid.org/0000-0001-9203-9954. 
Galician Roman epigraphy. To this end, a series of research experiences and practices developed for updating, revisiting and socialising the Roman inscriptions from such province are explained. The result is an online, open access, corpus which uses Structure from Motion Photogrammetry for recording the inscriptions and digital imaging techniques to better read the texts. The final goal is to reduce some of the negative effects that certain structural problems in Galicia, such as rural depopulation and demographic ageing, are causing on their preservation.

Keywords: Galicia; Roman epigraphy; cultural heritage; digital technologies; Web 3.0.

\section{INTRODUCCIÓN}

La provincia de Ourense es, desde el punto de vista patrimonial, la región de Galicia (España) con mayor número de bienes culturales de época romana (Fig. 1). Entre ellos ocupan un lugar destacado las inscripciones, que procuran importante información acerca de un amplio conjunto de aspectos de dicho periodo histórico (Bodel, 2001; d'Encarnação, 2006; Bruun y Edmondson, 2014). Sin embargo, la provincia de Ourense es, junto con la de Lugo, el territorio gallego con los índices más elevados de envejecimiento demográfico y despoblamiento rural (Hernández Borge, 2013; Saco Álvarez, 2013; Fernández Fernández y Meixide Vecino, 2013; Aldrey Vázquez y del Río Franqueira, 2014; Pazo Labrador y Moragón Arias, 2018), fenómenos que afectan también a numerosas regiones de España y la Europa mediterránea (Gómez-Limón, 2007; Tomassini y Lamura, 2009; Burillo Mozota, Burillo Cuadrado y Ruiz Budría, 2013; Pinilla Navarro y Sáez Pérez, 2016; FEMP, 2017a y 2017b; ESPON 2017) y cuyos efectos han sido calificados con expresiones tan elocuentes como «catástrofe demográfica», «extinción demográfica» y «demotanasia» (Pazo Labrador y Moragón Arias, 2014: 124-125).

Figura 1. Localización de la provincia de Ourense (Galicia, Noroeste de España).

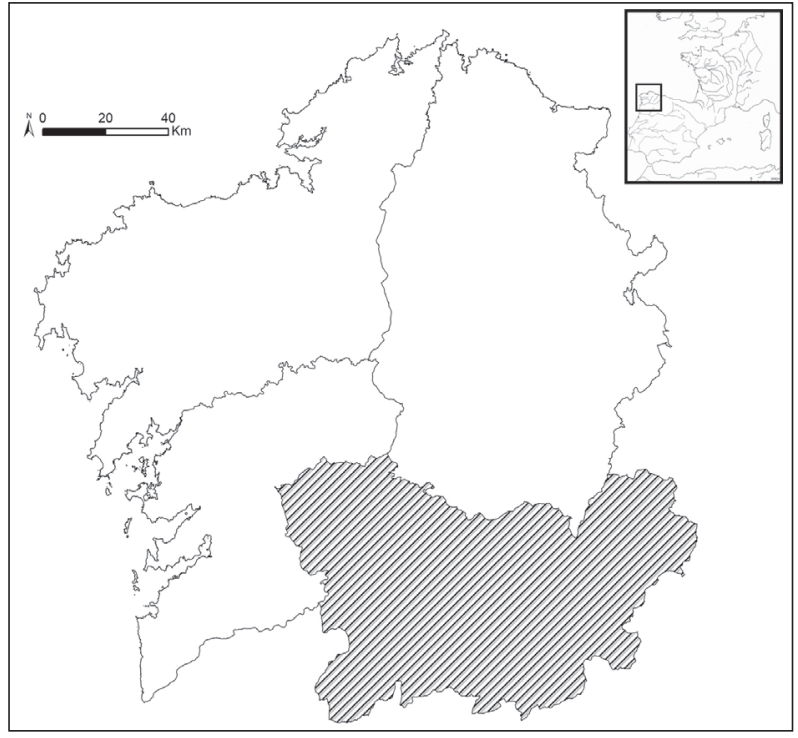


Con independencia de los factores multicausales que se encuentran en la raíz de estos procesos (Gómez-Limón, 2007: 27-31; Toxo-Asorei, 2016: 14-19), existe un creciente consenso en la investigación en torno a la idea de que tales fenómenos, responsables de la crisis del medio rural, afectan también de forma decisiva al patrimonio histórico y cultural (material e inmaterial) localizado en él, provocando en numerosas ocasiones su devaluación, abandono, sustracción y destrucción, tanto por acción como por omisión. Así lo han defendido autores como Acín Fanlo (2004: 20), Gómez-Limón (2007: 31-36), Fernández Fernández y Meixide Vecino (2013) y Sánchez Sánchez (2016: 67-69), para quienes la desaparición de los bienes patrimoniales lleva aparejada la inevitable pérdida de los rasgos identitarios y la memoria de las comunidades que los albergan.

Precisamente son los ciudadanos que habitan en su entorno los que, ante la merma de recursos materiales y servicios públicos, asumen el coste y la responsabilidad de su mantenimiento, custodia y conservación, si bien frecuentemente en condiciones precarias debido a una falta de experiencia y formación técnica necesarias (European Commission, 2014). Además, una vez que dicha población (generalmente de edad avanzada) deja de vivir en sus comunidades, desaparece con ella un importante y valioso caudal de información acerca de estos bienes, así como una red asistencial voluntariosa difícilmente restituible, convirtiéndose dicha labor (desde un punto de vista social y estructural) en insostenible, principalmente por la pérdida de interés y la falta de conocimiento acerca del valor histórico, económico y cultural de estos bienes (Sánchez Sánchez, 2016: 69 y 126).

Centrando la atención en el patrimonio ourensano, concretamente en las inscripciones romanas, la preservación, investigación y difusión de este tipo de recursos culturales no están libres de los problemas estructurales y patrimoniales previamente mencionados. Ubicadas mayoritariamente en entornos rurales con elevados índices de envejecimiento y despoblación, permanecen (en el mejor de los casos) en el interior de propiedades particulares y edificios eclesiásticos, sufriendo mayores problemas de conservación y conocimiento aquellas localizadas en entornos naturales al aire libre, sin ningún tipo de señalización ni medidas de protección. Todas ellas, sobre todo estas últimas, están expuestas a los avatares de la población que las acoge, quedando también afectadas por el paso del tiempo, las inclemencias meteorológicas y el olvido (Fig. 2. A, B).

Tomando en consideración estos problemas, la generalización de las tecnologías digitales y su aplicación en el campo de las Humanidades están permitiendo el desarrollo de proyectos que tienen por objeto un registro y análisis más precisos de los restos arqueológicos, entre ellos los epígrafes. En el ámbito del patrimonio cultural, esto está contribuyendo a la implementación de políticas de gestión y difusión innovadoras de los recursos, a partir de bases de datos relacionales cuya versatilidad favorece el almacenamiento y tratamiento informatizado de la información gráfica y textual, así como la realización de búsquedas especializadas y la socialización a gran escala en entornos Web. 

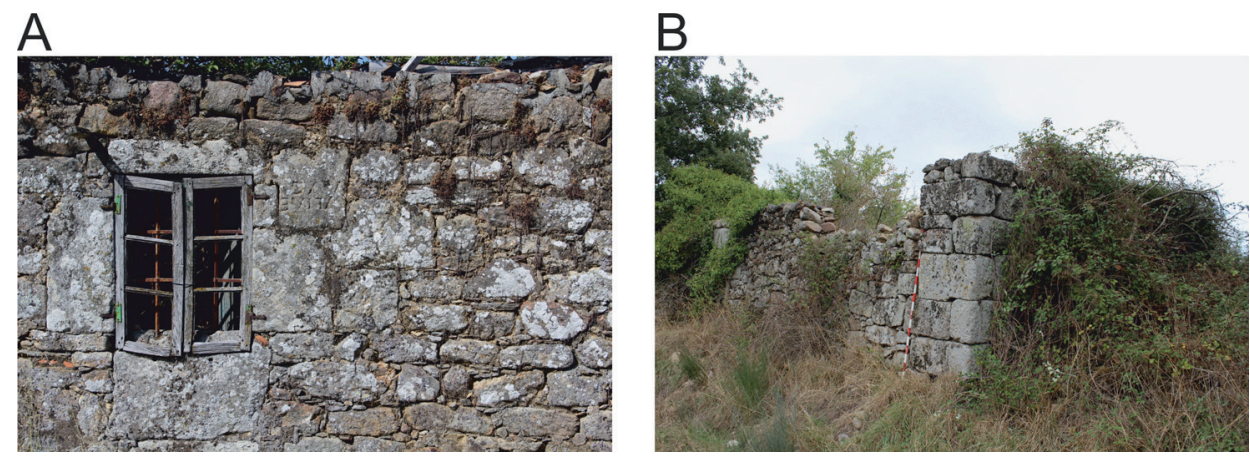

Figura 2. A: Inscripción honorífica de Castromao ( $A F \mathrm{I}^{2}$, 598: Santa María de Castromao, Celanova), actualmente empotrada en un muro de la antigua Casa Rectoral. B: Inscripción votiva de Ganade dedicada a Iuppiter Optimus Maximus (AF I², 38: San Bartolomeu de Ganade, Xinzo de Limia), reutilizada en un antiguo edificio abandonado.

Este aspecto está precisamente contemplado entre los objetivos y prioridades del Programa de Trabajo de Horizonte 2020 Europe in a changing world - Inclusive, innovative and reflective societies (European Commission 2017), del Plan de Trabajo de la Estrategia regional de especialización inteligente de Galicia (RIS3 Galicia) (Xunta de Galicia 2014) y del Programa Operativo FEDER Galicia 2014-2020 "Una manera de hacer Europa” (Xunta de Galicia, 2017). Respecto a este último, entre las estrategias y planes contemplados se encuentran dos relacionados con el empleo de las tecnologías digitales: uno destinado al fomento de la educación digital en el marco del sistema educativo gallego, y otro a la gestión digital del patrimonio cultural. El segundo, concretamente, trata de promover la aplicación de las Tecnologías de la Información y la Comunicación (TIC) en la catalogación, difusión y puesta en valor de los activos patrimoniales, constituyendo un elemento motriz de la dinamización económica y turística del territorio para favorecer su protección (Xunta de Galicia, 2017: 107-109 y 187). Los resultados de este plan, de acuerdo con la recomendación Towards an integrated approach to cultural heritage for Europe de la Comisión Europea (European Commission, 2014b), así como con la opinión de autores como Lincoln, Lindberg y Follet (2010) y Sánchez Sánchez (2016), supondría además una herramienta importante de desarrollo del medio rural.

Pero para alcanzar estos objetivos es necesario realizar antes un trabajo de identificación, registro, estudio y difusión de cada uno de los recursos patrimoniales disponibles. A este respecto, el proyecto "Epigraphica 3.0: Hacia la creación y diseño de un corpus digital de inscripciones latinas de la provincia de Ourense" está fundando las bases para contribuir, en el campo de la Epigrafía, a la consecución de los planes y objetivos mencionados. Especialmente relevante en este sentido es el corpus online y en open access que reunirá y publicará más de 200 inscripciones distribuidas por toda la provincia, una labor necesaria y urgente habida cuenta de la esclerotización y falta de recursos que padece la administración pública responsable de su documentación y preservación, así como de 
los efectos negativos derivados de los problemas endémicos que adolece este territorio en el marco de Galicia. De este modo, la provincia de Ourense, un "territorio periférico dentro de la periferia" (Pazo Labrador y Moragón Arias, 2018: 138), se convertirá en un laboratorio privilegiado de innovación en lo que a la gestión de una parte importante de sus recursos patrimoniales se refiere.

\section{LA EPIGRAFÍA ROMANA EN GALICIA}

La Comunidad Autónoma de Galicia posee una interesante y amplia colección de inscripciones romanas que han sido estudiadas y publicadas en una serie de artículos en revistas científicas, capítulos de libro y corpora epigráficos. Respecto a estos últimos, la sistematización y clasificación de los epígrafes han seguido criterios temáticos y administrativos modernos, centrados fundamentalmente en las provincias actuales: A Coruña, Lugo, Pontevedra y Ourense. En total, además de Corpus Inscriptionum Latinarum II. Inscriptiones Hispaniae Latinae (Hübner, 1869) y su suplemento (Hübner, 1892), las inscripciones romanas gallegas han sido reunidas en diez corpora distintos: Inscripciones romanas de Galicia (Bouza Brey et al., 1949-1968), Las estelas funerarias de Galicia en la época romana (Rodríguez Lage, 1974), Inscriptiones Romaines de la Province de Lugo (Arias Vilas, Le Roux, Tranoy, 1979), Aquae Flaviae. I. Fontes epigráficas (Rodríguez Colmenero, 1987), Corpus de inscripcións romanas de Galicia (Pereira Menaut, 1991; Baños Rodríguez, 1994), Corpus de inscripciones rupestres de época romana del cuadrante NW de la Península Ibérica (Rodríguez Colmenero, 1995), Aquae Flaviae. I. Fontes epigráficas da Gallaecia meridional interior (Rodríguez Colmenero, 1997), Miliarios e outras inscricións viarias romanas do noroeste hispánico (Conventos Bracarense, Lucense e Asturicense) (Rodríguez Colmenero, Ferrer Sierra, Álvarez Asorey, 2004), y Epigrafía romana de la provincia de Lugo (Gómez Vila, 2009).

En términos generales, el estado de la investigación epigráfica en Galicia es satisfactorio, sobre todo por el esfuerzo y trabajo de actualización realizado por revistas como Hispania Epigraphica, L'Année Épigraphique o Ficheiro Epigráfico. Sin embargo, el tiempo transcurrido desde la publicación de los citados corpora y las posibilidades ofrecidas por la expansión de las tecnologías digitales a través de la epigrafía latina (resultado de la evolución y ampliación de esta disciplina [Santiago Fernández, 2004; Ramírez Sánchez, 2005; Orlandi, Santucci y Liuzzo, 2017]), son motivos suficientes para proponer una puesta al día y una revisión completa del fondo epigráfico gallego de época romana. Si bien puede parecer, a tenor de los trabajos mencionados, que la investigación epigráfica en Galicia no plantea nuevos retos ni oportunidades (exceptuando la publicación de ejemplares inéditos o revisados), la aplicación de las tecnologías digitales a la epigrafía está sentando las bases de una renovación metodológica, técnica y de transferencia de conocimiento. Es precisamente en estos ámbitos en los que el proyecto Epigraphica 3.0 pretende contribuir a la investigación, difusión y preservación del patrimonio histórico y cultural gallego a través de una serie de prácticas concretas. 


\subsection{Retos y problemas de investigación}

El estudio de la epigrafía romana de Galicia, particularmente de aquella conservada en la provincia de Ourense, está afectado por un conjunto de problemas de investigación (además de por los de carácter endémico o estructural) que comportan verdaderas limitaciones a los métodos y técnicas tradicionales y al conocimiento epigráfico. Dichos problemas están relacionados, por un lado, con la localización y accesibilidad de los epígrafes, y, por otro, con el registro y legibilidad de los mismos, lo que supone un escollo evidente para la investigación histórica y su socialización.

En cuanto a la localización y accesibilidad de las inscripciones, la investigación epigráfica en este territorio está condicionada por un patrón de poblamiento de tipo disperso. Esto provoca que los trabajos de prospección sean más costosos de lo habitual en términos de tiempo y recursos económicos. A ello contribuye que un número importante de las inscripciones (tres cuartas partes del total) esté distribuido por todo el territorio provincial, careciendo además de las coordenadas geográficas necesarias para su precisa localización. Habida cuenta del mencionado fenómeno de despoblamiento rural que padece Ourense (con comarcas deshabitadas, donde no es posible disponer de la colaboración ciudadana para concretar su ubicación), esta circunstancia provoca que algunas inscripciones sean ilocalizables e irrecuperables. Por otro lado, en los casos en los que su localización es factible, existen problemas de accesibilidad derivados de su alojamiento en propiedades particulares o eclesiásticas cuyos titulares prohíben o restringen la entrada, así como de su reutilización como material constructivo en edificios modernos (Fig. 3. A, B).
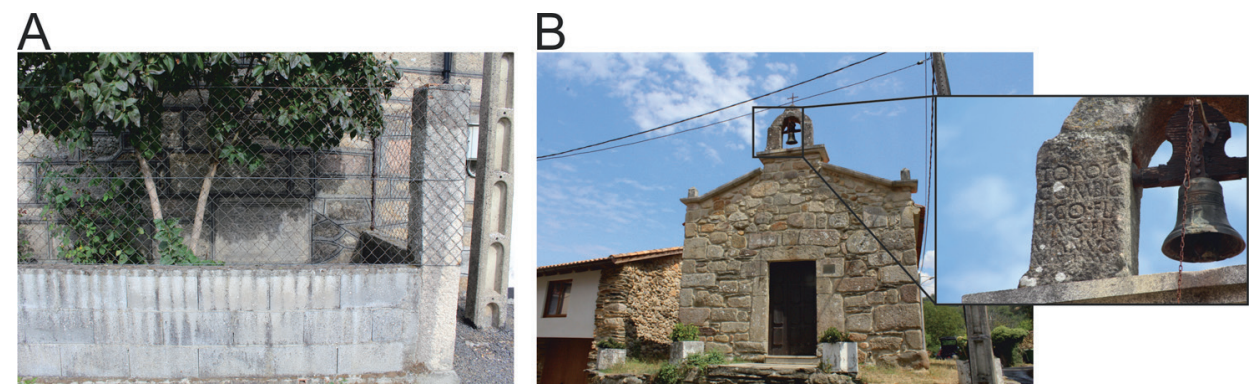

Figura 3. A: Placa funeraria de Manga de Abaixo dedicada a Marcus Iunius Robustus ( $A F \mathrm{I}^{2}$, 216: Lamas, Xinzo de Limia), actualmente conservada en el interior de una propiedad particular. B: Inscripción votiva de Pias ( $A F$ $\mathrm{I}^{2}$, 95: Castro Escuadro, Maceda), reutilizada en la espadaña de la capilla de San Pedro.

Respecto a legibilidad de los epígrafes, su exposición (sobre todo las de carácter rupestre) a los agentes erosivos y el paso del tiempo incrementa y acelera los procesos de degradación mecánica y biológica del granito en el que fueron realizadas: el desplacado, la desagregación granular o arenosa y la colonización por líquenes y musgos (Vicente 
Hernández, Delgado Rodrigues y Acevedo, 1996; Rivas Brea et al., 2002; Zambell et al., 2012; Freira-Lista y Fort, 2015) (Fig. 4. A, B, C). Esto dificulta el registro y análisis de las inscripciones, disminuyendo la legibilidad de los textos (muchos epígrafes son considerados ilegibles o ininteligibles) y motivando el empleo de determinadas técnicas y métodos de documentación arqueológica que comprenden la realización de calcos por frottage y la fotografía con luz rasante. En algunos casos, además, las fracturas y marcas del granito se confunden con los trazos de las letras, generando un elevado número de variantes de lectura. A su ilegibilidad contribuyen también las peculiaridades de la epigrafía romana gallega, afectada por el bajo nivel de destreza de los lapicidas que grabaron los textos, así como por un registro epigráfico sui generis en cuanto a nombres personales y divinidades, sin paralelos en otras regiones (Le Roux y Tranoy, 1973: 11). Esto determina que la lectura final de los epígrafes dependa sobremanera de la experiencia y percepción de los epigrafistas.
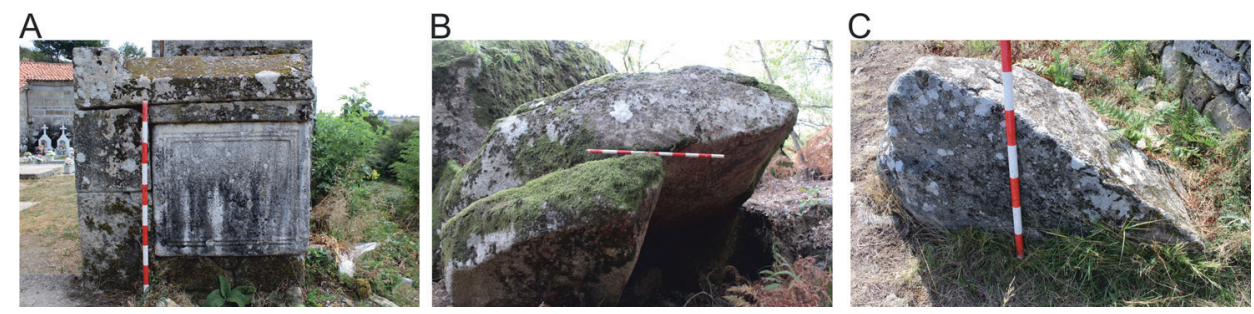

Figura 4. Efectos de la colonización biológica por líquenes y musgos. A: Placa funeraria de Escuadro $\left(A F \mathrm{I}^{2}\right.$, 207: Castro Escuadro, Maceda). B: Inscripción rupestre de San Salvador ( $A F \mathrm{I}^{2}$, 620: Santa Cristina de Vilariño, O Pereiro de Aguiar). C: Inscripción rupestre del Castro de San Cibrao de Las dedicada a Iuppiter (AF I², 55 : San Cibrao de Las, San Amaro).

\section{EPIGRAPHICA 3.0}

El proyecto de investigación «Epigraphica 3.0: Hacia la creación y diseño de un corpus digital de inscripciones latinas de la provincia de Ourense» tiene por objeto afrontar y superar los principales problemas de investigación que el estudio y la preservación del patrimonio epigráfico ourensano comportan. Financiado por la Consellería de Cultura, Educación e Ordenación Universitaria de la Xunta de Galicia (Plan I2C), y apoyado por la Universidade de Santiago de Compostela, el Consello da Cultura Galega, el Archivo Epigráfico de Hispania y el Centro de Supercomputación de Galicia, tiene como objetivo la aplicación de las tecnologías digitales en la investigación de la epigrafía romana ourensana en tres ámbitos: la localización y el registro documental, el análisis y la restitución textual, y la socialización del patrimonio y el conocimiento epigráficos.

En la consecución de este objetivo se han considerado las prácticas digitales y los resultados de investigación de proyectos como EAGLE (Orlandi, Giberti y Santuc- 
ci, 2014), i.Sicily (Prag, Chartrand y Cummings, 2017), Ashmolean Latin Inscriptions Project (Masséglia, 2014), Digital Epigraphy Toolbox (Bozia, Barmpoutis y Wagman, 2014), Epigraphia 3D (Ramírez Sánchez, García Sánchez y Giralt Soler, 2015) y Museo Virtual de Los Bañales (Andreu Pintado, 2018). En todos ellos, las bases de datos relacionales, la fotogrametría y el modelado 3D juegan un papel fundamental en el registro y la difusión de las inscripciones. Además de esto, Epigraphica 3.0 ha introducido por vez primera la aplicación de forma sistemática de una serie de técnicas de tratamiento de imagen digital en el proceso de análisis y restitución textual, lo que contribuirá a la elaboración de lecturas más seguras (Carrero Pazos y Espinosa Espinosa, 2018a y 2018b).

\subsection{Estadios metodológicos}

La consecución del proyecto está concretándose en una serie de estadios metodológicos relacionados con los tres ámbitos de investigación epigráfica. En cuanto a la localización y el registro epigráfico, Epigraphica 3.0 ha localizado y documentado la mayor parte de las inscripciones mediante prospección arqueológica. La finalidad es proporcionar su emplazamiento geográfico exacto mediante coordenadas. En esta labor resultaron imprescindibles los trabajos previos de documentación desarrollados en el Archivo Epigráfico de Hispania, el Museo Arqueológico Provincial de Ourense y la Dirección Xeral do Patrimonio Cultural de la Xunta de Galicia. En dichos centros se llevó a cabo la consulta de bibliografía especializada (particularmente el vaciado de la segunda edición del corpus impreso Aquae Flaviae. I. Fontes epigráficas da Gallaecia meridional interior [Rodríguez Colmenero, 1997]) e información catalográfica. Una vez ha sido hallada cada inscripción, se practica una autopsia completa in situ mediante el empleo de una ficha de campo (Tabla I) y el correspondiente levantamiento fotogramétrico según los principios de solape publicados (Agisoft, 2012; Verhoeven, 2011: 71; Remondino, 2014).

Respecto al análisis y la restitución textual, del conjunto de fotografías realizadas se eligen aquellas que permiten la creación de un modelo 3D preciso de cada inscripción mediante Agisoft Photoscan según los principios de stereo matching (Plets et al., 2012) (Fig. 5. A, B , C). Una vez dicho modelo ha sido creado, orientado y escalado, se aplica una serie de técnicas de postprocesado de imagen digital mediante MeshLab con la finalidad de identificar los trazos correspondientes al texto, especialmente en el caso de epígrafes seriamente deteriorados. Entre las técnicas aplicadas hay que mencionar la iluminación artificial del modelo 3D sin textura, el radiance scaling, la coloración de la curvatura, la comparación de mallas y el sombreado exagerado, todas ellas conocidas por su aplicación a diferentes vestigios arqueológicos (Carrero Pazos, Vilas Estévez y Vázquez Martínez, 2017; Carrero Pazos y Espinosa Espinosa, 2018a, 2018b) (Fig. 6. A, B, C, D, E). Una vez toda la información gráfica ha sido procesada y analizada, se realiza una propuesta de restitución textual siguiendo los principios del sistema Leiden de signos diacríticos, cumplimentándose con toda la información disponible una base de datos relacional mediante el gestor MariaDB, que constituye la fuente del corpus epigráfico alojado en la web www.epigraphica30.com. 
Tabla I. Ficha de campo diseñada para Epigraphica 3.0.

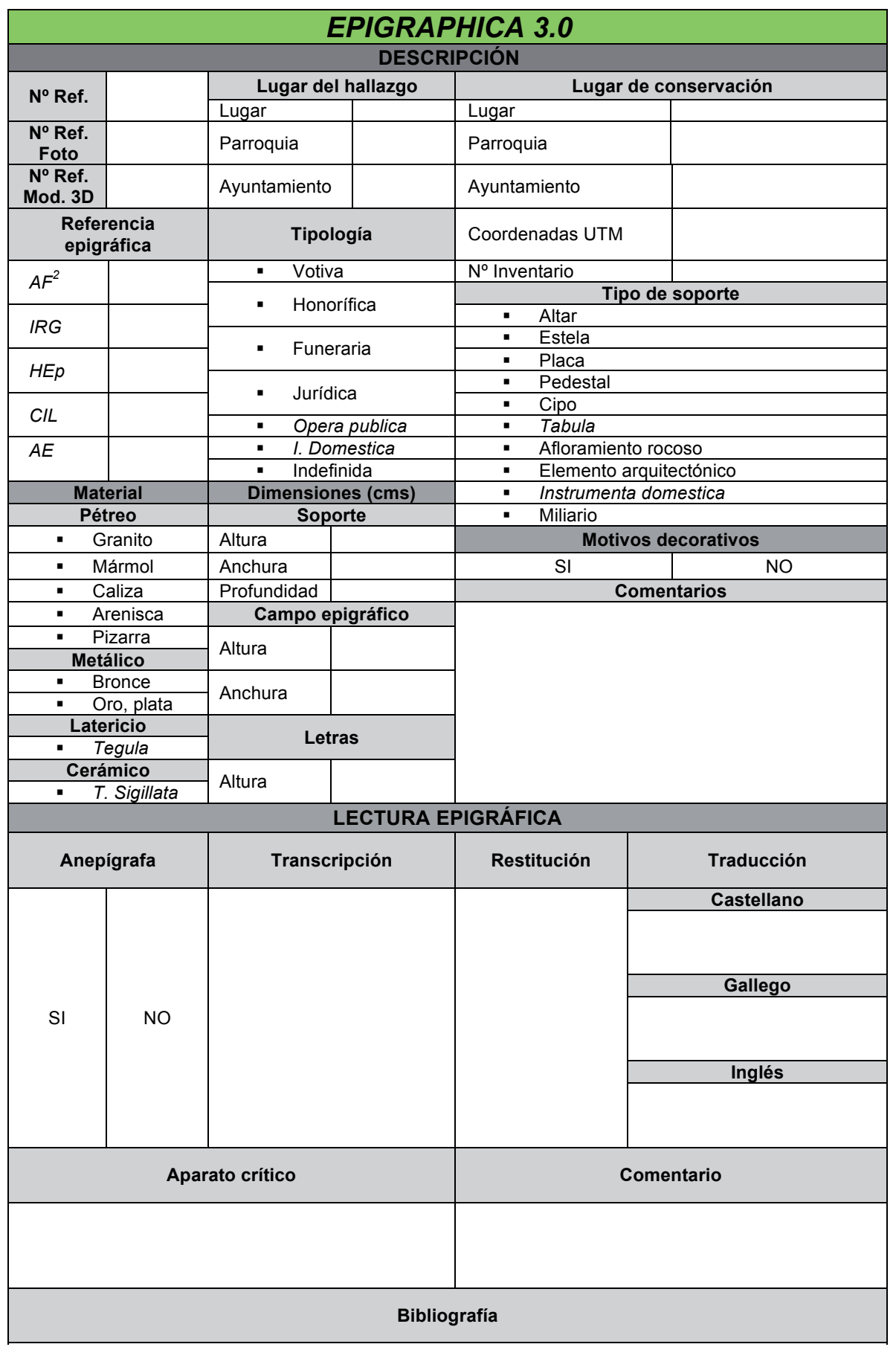


Tabla I (continuación)

\begin{tabular}{|c|c|c|c|}
\hline \multicolumn{4}{|c|}{ EPIGRAPHICA 3.0 } \\
\hline \multicolumn{3}{|c|}{ MODELADO 3D } \\
\hline Modelo & Tiempo de exposición & $\begin{array}{c}\text { Número de } \\
\text { imágenes }\end{array}$ & Caras modelo 3D \\
\hline Megapíxeles & Velocidad ISO & Calidad & Vértices modelo 3D \\
\hline Resolución & $\begin{array}{c}\text { Compensación de } \\
\text { exposición }\end{array}$ & $\begin{array}{c}\text { Solapamiento } \\
\text { efectivo }\end{array}$ & Textura \\
\hline & Distancia focal & $\begin{array}{c}\text { Error de } \\
\text { reproyección }\end{array}$ & $\begin{array}{c}\text { Tiempo de procesado } \\
\text { (total) }\end{array}$ \\
\hline Punto F & & & \\
\hline
\end{tabular}
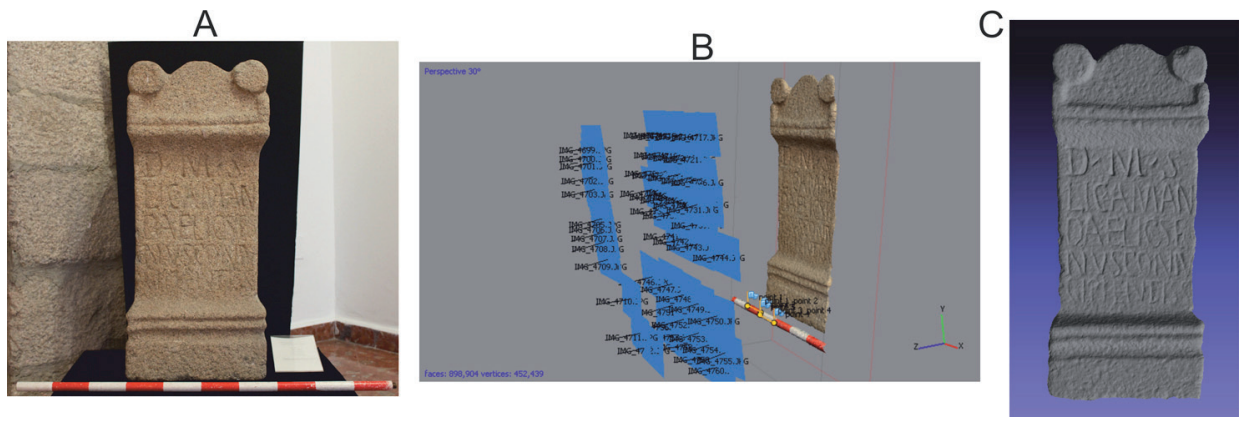

Figura 5. Proceso de registro fotogramétrico. A: Inscripción funeraria de Licinia Amanda (HEp 2, 1990, 575 : Ourense, Ourense). B: Orientación y escalado del modelo 3D. C: Resultado final del modelo 3D.

En relación con la socialización del patrimonio y el conocimiento epigráficos, el proyecto pone al servicio de la comunidad investigadora y del público en general toda la documentación de cada inscripción (tanto gráfica como textual) con el objetivo de difundir y preservar el fondo epigráfico ourensano de época romana. Para ello, además de facilitar la consulta e interacción con la información mediante un buscador avanzado, un visor cartográfico y un visualizador de modelos 3D, el corpus prevé la descarga de la información mediante paquetes de datos en formato PLY y fichas epigráficas como archivos PDF 3D. 

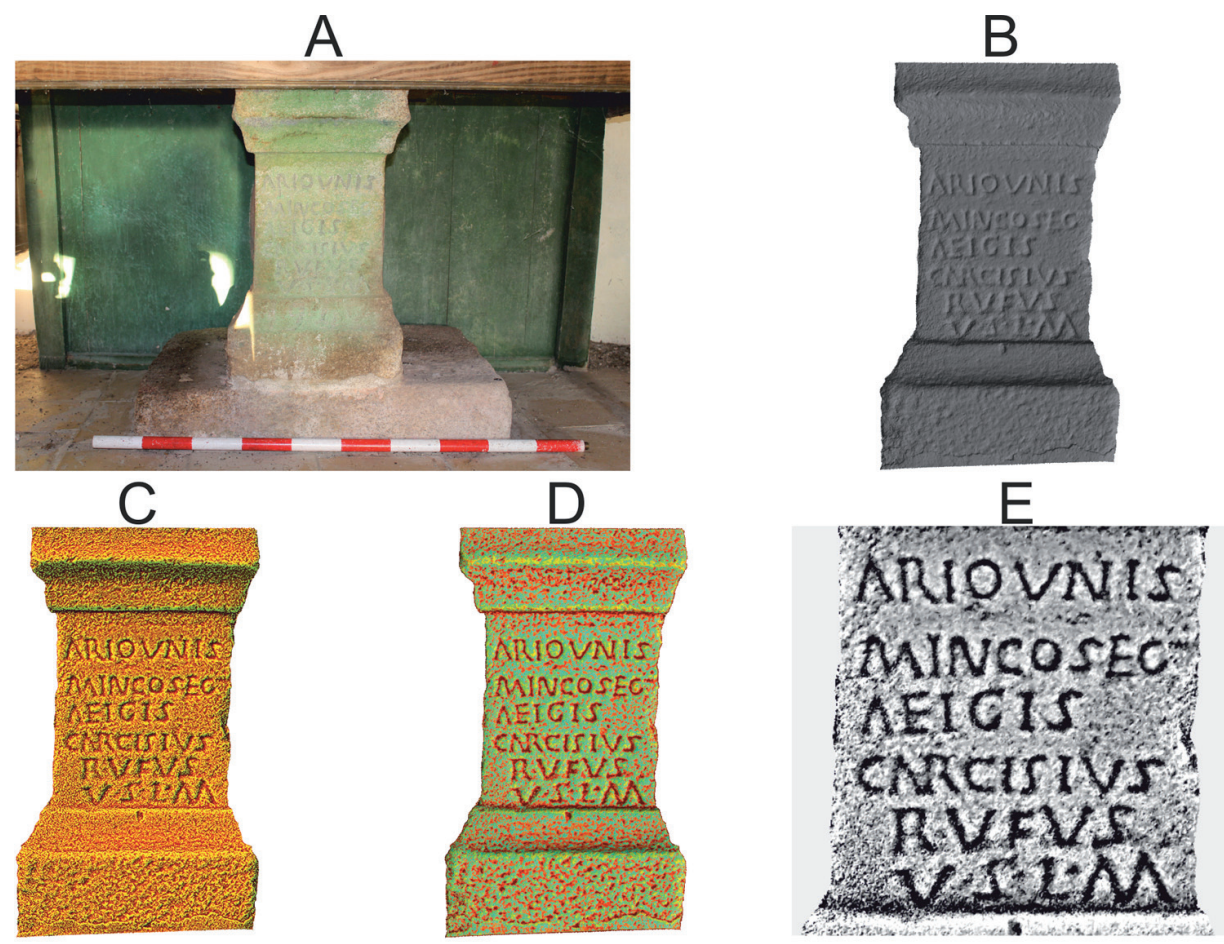

Figura 6. Resultados de los análisis de la inscripción votiva de Nocelo $\left(A F \mathrm{I}^{2}, 159\right.$ : Sobreganade, Porqueira) mediante técnicas de postprocesado. A: Fotografía actual de la inscripción. B: Modelo 3D sin textura con luces artificiales. C: Radiance scaling con doble esfera. D: Comparación de mallas. E: Sombreado exagerado.

\subsection{Principales resultados y contribuciones}

Teniendo en mente los efectos negativos que sobre el patrimonio histórico y cultural provocan el despoblamiento rural y el envejecimiento demográfico (específicamente en la provincia de Ourense), los objetivos, prioridades y recomendaciones formuladas por las administraciones autonómica, nacional y europea, y los problemas de investigación en el ámbito de la epigrafía gallega de época romana, los principales resultados y contribuciones de Epigraphica 3.0 son:

1) La localización exacta de cada inscripción mediante el empleo de coordenadas UTM. Habida cuenta de los problemas de localización y accesibilidad, el proyecto busca procurar al investigador y al público no especializado referencias geográficas precisas para cada testimonio epigráfico. Esta información es ofrecida mediante un visor cartográfico creado con la API de Google Maps. Además, en los casos en los que es posible, se ofrecen tanto el lugar del hallazgo como el lugar de conservación mediante su topónimo antiguo y moderno. Para ello se emplea, siguiendo la recomendación del consorcio 
EAGLE con el objetivo de armonizar los datos y evitar problemas de homonimia, la información disponible en los portales Pleiades, Trismegistos y Geo Names.

2) La aplicación de técnicas no invasivas en las operaciones de registro, documentación y lectura del campo epigráfico. Frente a los métodos y técnicas de registro tradicionales caracterizados, en algunos casos, por su naturaleza invasiva, el proyecto utiliza la fotogrametría Structure from Motion (SfM) y el tratamiento de imagen digital para analizar y restituir los textos. Esto permite preservar la integridad de los epígrafes, garantizando al mismo tiempo unos resultados mucho más precisos y seguros (Mittica, Pellegrino y Rocco, 2014; Pires, Martínez Rubio, Elorza Arana, 2015: 416-417). Asimismo, como han señalado autores como Mittica, Pellegrino y Rocco (2014: 402), la documentación en $3 \mathrm{D}$ tiene entre sus beneficios «the ability to virtually measure, rotate, zoom or illuminate the object, thus replicating all possible points of view; the convenience of data storage on a digital support, that does not need space and is not subjected to degradation; the ease of duplicating and sharing».

3) La creación de un corpus online y en open access en continua actualización. La principal contribución de Epigraphica 3.0 es la construcción de un corpus epigráfico digital a partir de una base de datos relacional según el concepto de digital library (Babeu, 2011: 8; Carloni y Guercio, 2014; Bozia, 2017). Dicho corpus facilitará la búsqueda, consulta e interacción con la información gráfica y textual por parte del usuario. Si bien inicialmente estará disponible en gallego y castellano, a medio plazo se prevé desarrollar una versión en inglés. Entre sus prestaciones destacan la adaptabilidad a cualquier dispositivo electrónico, la adecuación de los criterios de búsqueda a las necesidades de los usuarios, la interactividad con la información mediante un visor cartográfico y un visualizador de modelos 3D (3D Heritage Online Presenter, Isti-CNR de Pisa: Potenziani et al., 2015), y la posibilidad de descargar la información de acuerdo con los principios de reproducible research y open science. Además, para facilitar la recuperación y el tratamiento de la información, el corpus incluye referencias cruzadas y enlaces a bases de datos online como Hispania Epigraphica. También, a diferencia de otros corpora, proporciona la traducción de cada inscripción en varias lenguas, haciendo de este modo más accesible la información epigráfica. La idoneidad de esta práctica se ha discutido en reuniones celebradas por EAGLE (Bigi, 2014), y se incluye entre los objetivos de proyectos como I.Siciliy (Prag, Chartrand, Cummings, 2017: 91).

\section{CONCLUSIONES}

Como se ha tratado de mostrar en este trabajo, Epigraphica 3.0 es un proyecto piloto, de orientación epigráfica y naturaleza interdisciplinar, surgido en el marco de una concienciación creciente en la administración pública sobre la necesidad de conocer, preservar y poner en valor los distintos recursos del patrimonio histórico y cultural, como una forma de generar riqueza social y económica. Por ello, en el ámbito de la epigrafía 
ourensana, Epigraphica 3.0 ha apostado por un empleo crítico de las tecnologías digitales con el objetivo de optimizar los diferentes estadios metodológicos del proceso de investigación epigráfica. Sobre estas bases, a partir de la revisión y actualización del corpus epigráfico de la provincia de Ourense, Epigraphica 3.0 está afrontando satisfactoriamente un doble reto: 1) la disminución de los efectos negativos que fenómenos estructurales como el despoblamiento rural y el envejecimiento demográfico tienen sobre el conocimiento y la conservación del patrimonio epigráfico, 2) la formulación y propuesta de soluciones a los problemas de investigación que plantea el estudio de la epigrafía romana en Galicia.

Respecto al primero de estos retos, Epigraphica 3.0 aspira a convertirse en un referente investigador para una gestión innovadora de los recursos patrimoniales de Galicia (concretamente de los testimonios epigráficos romanos) a través de una doble estrategia: 1) la localización y el registro de cada inscripción como un medio para garantizar su conocimiento histórico y preservación documental, 2) la socialización de este tipo de bienes culturales que acusa una importante desatención por parte de la ciudadanía y las administraciones públicas, debido al desconocimiento existente acerca de su importancia documental y valor histórico. De esta forma, el corpus resultante (online y en open access) se configura como una poderosa herramienta para la toma de decisiones científicas, socioeconómicas y culturales.

En cuanto al segundo de los retos, Epigraphica 3.0 espera constituirse en un referente técnico y metodológico para la investigación epigráfica en Galicia y el resto de la Península Ibérica, debido a la explotación de los beneficios y las posibilidades de las tecnologías digitales en el ámbito de la Epigrafía. Concretamente, por las ventajas ofrecidas por el procesamiento digital de la información textual y gráfica, en tres áreas: 1) la localización y el registro documental a través de la georreferenciación, el levantamiento fotogramétrico y la grabación y el tratamiento de los datos mediante una base de datos relacional, 2) el análisis y la restitución textual a través del modelado 3D y el procesamiento de imagen digital, 3) la accesibilidad y socialización del patrimonio y conocimiento epigráficos mediante una página web programada en varias lenguas, que permite la búsqueda especializada, consulta interactiva y descarga selectiva de la información.

Finalmente, Epigraphica 3.0 no busca solamente promover en la ciudadanía un mayor conocimiento, interés y compromiso con la preservación y puesta en valor de la epigrafía romana ourensana, sino también fijar unas bases documentales sólidas y objetivas para el desarrollo de futuras investigaciones sobre el territorio gallego en época romana. En este sentido, el tratamiento informatizado y el análisis estadístico de la información epigráfica en formato digital puede llegar a permitir la identificación de talleres y paisajes epigráficos, así como la consideración de determinados fenómenos históricos y culturales del proceso romanizador. En definitiva, Epigraphica 3.0 trasciende la materialidad del testimonio epigráfico para ofrecer a las administraciones, los investigadores y el público no especializado un conocimiento más preciso de la realidad patrimonial y la información histórica y cultural que atesora. 


\section{BIBLIOGRAFÍA}

Acín Fanlo, J. L. (2004): "La despoblación como cuestión científica y humana”, Revista Ripacurtia 2. Dossier: La despoblació a la Ribagorça, pp. 15-32.

Agisoft L.L.C. (2012): Agisoft PhotoScan user manual: Professional Edition, Version 0.8.3, Moscow, AgiSoft LLC.

Aldrey Vázquez, J. A. y del Río Franqueira, D. (2014): “Crónica de un envejecimiento anunciado: Galicia 2015-2030”, en López Gay, A., Rofo, F., Solsona, M., Andújar, A., Cruz, J., Iglesias, R., Feria, J. M., Vahí, A. (eds.), Cambio demográfico y socio territorial en un contexto de crisis, Sevilla, Grupo de Población de la AGE, pp. 69-82.

Andreu Pintado, F. J. (2018): “Museo histórico y arqueológico virtual 'Los Bañales': fotogrametría 3D al servicio de la puesta en valor del patrimonio arqueológico y de la formación universitaria", Cuadernos del Marqués de San Adrián Número Extraordinario, pp. 25-32.

Arias Vilas, F., Le Roux, P. y Tranoy, A. (1979): Inscriptiones Romaines de la Province de Lugo, Paris, De Boccard.

Babeu, A. (2011): “Rome Wasn't Digitized in a Day”: Building a Cyberinfrastructure for Digital Classics, Washington, Council on Library and Information Resources.

Baños Rodríguez, G. (1994). Corpus de inscripcións romanas de Galicia. Vol. II: Provincia de Pontevedra (CIRG II), Santiago de Compostela, Consello da Cultura Galega.

Bigi, F. (2014): "Towards an EAGLE Standard in Translating Inscriptions", en S. Orlandi, R. Santucci, V. Casarosa y P. M. Liuzzo (eds.), Information Technologies for Epigraphy and Cultural Heritage. Proceedings of the First EAGLE International Conference, Rome, Sapienza Università Editrice, pp. 167-178.

Bodel, J. (ed.) (2001): Epigraphic Evidence: Ancient History from Inscriptions, London, Routledge.

Bouza Brey, F., d’Ors, Á., Castillo, Á. del, Vázquez Saco, F., Vázquez Seijas, M., Figueira Valverde, J., Álvarez Vlázquez, J. M. a, Lorenzo Fernández, J. (1949-1968): Inscripciones romanas de Galicia, Santiago de Compostela, Instituto de Estudios Gallegos 'Padre Sarmiento'.

Bozia, E. (2017): "Assessing the Role of Digital Libraries of Squeezes in Epigraphic Studies. Digitization, visualization, and metadata", en S. Orlandi, R. Santucci, F. Mambrini y P. M. Liuzzo (eds.), Digital and Traditional Epigraphy in Context. Proceedings of the EAGLE 2016 International Conference, Rome, Sapienza Università Editrice, pp. 405-409.

Bozia, E., Barmpoutis, A., y Wagman, R. S. (2014): “Open-Access Epigraphy. Electronic Dissemination of 3D-digitized Archaeological Material”, en S. Orlandi, R. Santucci, V. Casarosa y P. M. Liuzzo (eds.), Information Technologies for Epigraphy and Cultural Heritage. Proceedings of the First EAGLE International Conference, Rome, Sapienza Università Editrice, pp. 421-435. 
Brunn, Chr. y Edmondson, J. (2014): The Oxford Handbook of Roman Epigraphy, Oxford, Oxford University Press.

Burillo Mozota, M. P., Burillo Cuadrado, F. y Ruiz Budría, E. (2013): Serranía Celtibérica (España): un proyecto de desarrollo rural para la Laponia del Mediterráneo, Zaragoza, Instituto Celtiberia de Investigación y Desarrollo Rural.

Carloni, C., y Guercio, M. (2014): "Livelli descrittivi, relazioni e contesti di produzione nella Sapienza Digital Library", en F. Ciotti (ed.), Digital Humanities: progetti italiani ed esperienze di convergenza multidisciplinare. Atti del convegno annuale dell'Associacione per l'Informatica Umanistica e la Cultura Digitale (AIUCD), Firenze, 13-14 dicembre 2012, Rome, Sapienza Università Editrice, pp. 51-70.

Carrero Pazos, M., y Espinosa Espinosa, D. (2018a): "Back to Basics. A Non-Photorealistic Rendering Method for the Analysis of Texts from 3D Roman Inscriptions". Antiquity 92(364), pp. 1-7.

Carrero Pazos, M., y Espinosa Espinosa, D. (2018b): “Tailoring 3D Modeling Techniques for Epigraphic Texts Restitution. Case Studies in Deteriorated Roman Inscriptions", Digital Applications in Archaeology and Cultural Heritage 10, pp. 1-12.

Carrero Pazos, M., Vilas Estévez, B., y Vázquez Martínez, A. (2017): “Making Visible the Invisible: Low Cost Methodologies for the Study of Ancient Carvings", en V. Mayoral Herrera, C. Parcero-Oubiña y P. Fábrega-Álvarez (eds.), Archaeology \& Geomatics. Harvesting the Benefits of 10 Years of Training in the Iberian Peninsula (2006-2015), Leiden, Sidestone Press, pp. 73-90.

d'Encarnação, J. (2006): Epigrafia. As Pedras que Falam, Coimbra, Imprensa da Universidade de Coimbra.

ESPON (2017): "Shrinking rural regions in Europe. Towards smart and innovative approaches to regional development challenges in depopulating rural regions", European Observation Network for Territorial Development and Cohesion.

European Commission (2014): Towards an integrated approach to cultural heritage for Europe, European Commission.

European Commission (2017): “13. Europe in a changing world - Inclusive, innovative and reflective societies", in Horizon 2020. Work Programme 2018-2020, European Commission Decision C(2017)7124 of 27 October 2017.

FEMP (2017a): "Población y despoblación en España 2016. El 50\% de los municipios españoles, en riesgo de extinción", Comisión de Despoblación, Federación Española de Municipios y Provincias.

FEMP (2017b): "Listado de medidas para luchar contra la despoblación en España”, Comisión de Despoblación, Federación Española de Municipios y Provincias.

Fernández Fernández, M. y Meixide Vecino, A. (2013): "Declive demográfico en Galicia y territorio: el diseño de políticas públicas en un espacio complejo y heterogéneo", en Escola Galega de Administración Pública (ed.), O reto demográfico de Galicia (Xornadas 1), Santiago de Compostela, Xunta de Galicia, pp. 59-80. 
Freire-Lista, D. M. y Fort, R. (2015): “Origen y evolución de desplacados y descamados en el granito de la Plaza Mayor de Madrid”, en M. a Moreno Oliva, M. A. RogerioCandelera, J. T. López Navarrete y V. Hernández Jolín (eds.), Estudio y Conservación del Patrimonio Cultural. Actas, Málaga, 16-19 Noviembre, 2015, Málaga, Red de Ciencia y Tecnología para la Conservación del Patrimonio Cultura - Universidad de Málaga, pp. 188-191.

Gómez-Limón, J. A. (2007): "Percepción pública del problema de la despoblación del medio rural en Castilla y León”, Ager. Revista de Estudios sobre Despoblación y Desarrollo Rural 6, pp. 9-60.

Gómez Vila, J. (2009): Epigrafía romana de la provincia de Lugo, Lulu Press, Londres.

Hernández Borge, J. (2013): Galicia, unha población avellentada. Santiago de Compostela: Servicio de Publicacións e Intercambio Científico de la Universidade de Santiago.

Hübner, E. (ed.) (1869): Corpus Inscriptionum Latinarum II. Inscriptiones Hispaniae Latinae, Berlin.

Hübner, E. (ed.) (1892): Corpus Inscriptionum Latinarum II. Inscriptiones Hispaniae Latinarum Supplementum, Berlin.

Le Roux, P., y Tranoy, A. (1973): "Notes d'épigraphie romaine de Galice", Cuadernos de Estudios Gallegos 28(85), pp. 221-234.

Lincoln, B., Lindberg, J. y Follet, J. A. (2010): Heritage-Based Rural Development Forum Journal, Winter 2010, Washington, DC, National Trust for Historic Preservation.

Masséglia, J. (2014): “The Ashmolean Latin Inscriptions Project (AshLI). Bringing epigraphic research to museum visitors and schools", en S. Orlandi, R. Santucci, V. Casarosa y P. M. Liuzzo (eds.), Information Technologies for Epigraphy and Cultural Heritage. Proceedings of the First EAGLE International Conference, Rome, Sapienza Università Editrice, pp. 221-232.

Mittica, D., Pellegrino, M., y Rocco, A. (2014): “Low-cost Structure from Motion Technology", en S. Orlandi, R. Santucci, V. Casarosa y P. M. Liuzzo (eds.), Information Technologies for Epigraphy and Cultural Heritage. Proceedings of the First EAGLE International Conference, Rome, Sapienza Università Editrice: 401-420.

Orlandi, S., Giberti, L., y Santucci, R. (2014): "EAGLE - Europeana network of Ancient Greek and Latin Epigraphy: Making the ancient inscriptions accessible", Lexicon Philosophicum. International Journal for the History of Texts and Ideas 2, pp. 315326.

Orlandi, S., Santucci, R., Mambrini, F., y Liuzzo, P. M. (eds.) (2017): Digital and Traditional Epigraphy in Context. Proceedings of the EAGLE 2016 International Conference, Rome, Sapienza Università Editrice.

Pazo Labrador, A. J. y Moragón Arias, M. ${ }^{a}$ P. (2018): “El despoblamiento en Galicia: la visualización de la "catástrofe", Ager. Revista de Estudios sobre Despoblación y Desarrollo Rural 24, pp. 123-154. 
Pereira Menaut, G. (1991): Corpus de inscripcións romanas de Galicia. Vol. I: Provincia de A Coruña (CIRG I), Santiago de Compostela, Consello da Cultura Galega.

Pinilla Navarro, V. y Sáez Pérez, L. A. (2016): La despoblación rural en España: génesis de un problema y políticas innovadoras, Zaragoza, Centro de Estudios sobre Despoblación y Desarrollo de Áreas Rurales.

Pires, H., Martínez Rubio, J., y Elorza Arana, A. (2015): “Techniques for Revealing 3D Hidden Archaeological Features: Morphological Residual Models as Virtual-Polynomial Texture Maps", The International Archives of the Photogrammetry, Remote Sensing and Spatial Information Sciences, Volume XL-5/W4, pp. 415-421.

Plets, G., Verhoeven, G., Cheremisin, D., Plets, R., Bourgeois, J., Stichelbaut, B., Gheyle, W., y De Reu, J. (2012): “The deteriorating preservation of the Altai rock art: assesing three dimensional image based modelling in rock art research and management", Rock Art Research 29(2), pp. 139-156.

Potenziani, M., Callieri, M., Dellepiane, M., Corsini, M., Ponchio, F., y Scopigno, R. (2015): “3DHOP: 3D Heritage Online Presenter”, Computers \& Graphics 52, pp. $129-141$.

Prag, J., Chartrand, J., y Cummings, J. (2017): "I.Sicily. An EpiDoc Corpus of Ancient Sicily”, en S. Orlandi, R. Santucci, F. Mambrini y P. M. Liuzzo (eds.), Digital and Traditional Epigraphy in Context. Proceedings of the EAGLE 2016 International Conference, Rome, Sapienza Università Editrice, pp. 83-96.

Ramírez Sánchez, M. (2005): "El concepto de Epigrafía: consideraciones sobre la necesidad de su ampliación, cincuenta años después”, Signo: revista de historia de cultura escrita 15 , pp. 47-76.

Ramírez Sánchez, M., García Sánchez, M., y Giralt Soler, S. (2015): “Epigraphia 3D. Un proyecto de innovación científica en la divulgación del patrimonio epigráfico de Hispania”, Epigraphica. Periodico Internazionale di Epigrafia 77(1-2), pp. 371-396.

Remondino, F. (2014): "Photogrammetry. Basic Theory", en F. Remondino y S. Campana (eds.), 3D recording and modelling in Archaeology and Cultural Heritage, Oxford, BAR International Series 2598, pp. 63-72.

Rivas Brea, T., Prieto Lamas, B., Lantes, O., y Silva, B. (2002): “Grado de meteorización y formas de alteración de las rocas de construcción de megalitos gallegos", Nova Acta Científica Compostelana (Bioloxía) 12, pp. 203-217.

Rodríguez Colmenero, A. (1987): Aquae Flaviae. I. Fontes epigráficas, Chaves, Câmara Municipal de Chaves.

Rodríguez Colmenero, A. (1995): "Corpus de inscripciones rupestres de época romana del cuadrante NW de la Península Ibérica”, en A. Rodríguez Colmenero, L. Gasperini (eds.), Saxa Scripta (Inscripciones en roca). Actas del Simposio Internacional Ibero-Itálico sobre epigrafía rupestre, Santiago de Compostela y Norte de Portugal, 29 de junio a 4 de julio de 1992, Sada, A Coruña, Ediciós do Castro, pp. 117-260.

Rodríguez Colmenero, A. (1997): Aquae Flaviae. I. Fontes epigráficas da Gallaecia meridional interior, Chaves, Câmara Municipal de Chaves. 
Rodríguez Colmenero, A., Ferrer Sierra, S. y Álvarez Asorey, R. D. (2004): Miliarios e outras inscricións viarias romanas do noroeste hispánico (Conventos Bracarense, Lucense e Asturicense), Santiago de Compostela, Consello da Cultura Galega.

Rodríguez Lage, S. (1974): Las estelas funerarias de Galicia en la época romana, Ourense, Instituto de Estudios Orensanos "Padre Feijoo".

Saco Álvarez, A. (2010): "Desarrollo rural y despoblación en Galicia: escenarios y desarrollos de futuro", Ager. Revista de Estudios sobre Despoblación y Desarrollo Rural 9, pp. 9-126.

Sánchez Sánchez, A. (2016): Behind the Ecce Homo: Rural Development Policy and the Effects of Depopulation on the Preservation of Spanish Heritage (Unpublished Master's Thesis), Columbia University, Graduate School of Architecture, Planning and Preservation.

Santiago Fernández, J. de (2004): "La epigrafía: evolución conceptual y metodológica". Documenta \& Instrumenta 1, pp. 203-220.

Tomassini, C. y Lamura, G. (2009): "Population Ageing in Italy and Southern Europe", in P. Uhlenberg (ed.), International Handbook of Population Aging, vol. 1, Springer, Dordrecht, pp. 69-89.

Toxo-Asorei, R. (2016): “Existe un 'problema demográfico' en Galicia?”, Revista AGALI 6, pp. 6-23.

Verhoeven, G. (2011): “Taking computer vision aloft - archaeological three-dimensional reconstructions from aerial photographs with photoscan", Archaeological Prospection 18, pp. 67-73.

Vicente Hernández, M. A., Delgado Rodrigues, J., y Acevedo, J. (eds.) (1996). Degradation and conservation of granitic rocks in monuments. Proceedings of the EC workshop held in Santiago de Compostela (Spain) on 28-30 November 1994. Protection and Conservation of the European Cultural Heritage. Research Report 5, European Commission: Brussels.

Xunta de Galicia (2014): Estrategia regional de especialización inteligente de Galicia, Santiago de Compostela, Xunta de Galicia.

Xunta de Galicia (2017): Programa Operativo FEDER Galicia 2014-2020 “Una manera de hacer Europa”, Xunta de Galicia.

Zambell, C. B., Adams, J. M., Gorring, M. L., y Schwartzman, D.W. (2012): "Effect of lichen colonization on chemical weathering of hornblende granite as estimated by aqueous elemental flux", Chemical Geology 291, pp. 166-174. 